\title{
Chronic cough following cardiac transplantation: vagal Mitempfindung?
}

\author{
R R Hammond, G C Ebers
}

\begin{abstract}
Since operation a cardiac transplant recipient has suffered from chronic, nonproductive but intense coughing spells triggered by stimulation of the right external ear. This demonstrates the unusual phenomenon of acquired aberrant sensory referral.
\end{abstract}

$(\mathcal{F}$ Neurol Neurosurg Psychiatry 1992;55:723-724)

\section{Case report}

This fifty seven year old retired mechanic was investigated for a chronic dry cough which had persisted since cardiac transplant surgery six months earlier for end-stage ischaemic cardiomyopathy. Before the operation the patient had no cough. Postoperatively he had hoarseness thought to be secondary to intubation. This lasted for two weeks and resolved. On the third postoperative day the patient developed a cough productive of yellow sputum. Chest radiographs showed left lower lobe atelectasis and a small left pleural effusion. The productive cough and pleural effusion cleared quickly and the left lower lobe changes cleared radiographically within three weeks. $\mathrm{He}$ was eventually discharged home six weeks after the transplant surgery with a persistent dry cough which had developed shortly before discharge. His paroxysms of dry cough were accompanied by a tickling sensation in his larynx, and gagging with some episodes. Shortly after discharge the patient realised that by manually stimulating his right ear, external auditory canal, or a small patch of skin behind the right ear an irritating tickling sensation in his larynx would be followed shortly by a severe paroxysm of dry cough and occasionally gagging. He found that lying on his right side with his head in contact with an object (such as a pillow) would also bring on a laryngeal irritation and a paroxysm of dry cough. Before his transplant he had never been able to produce anything remotely similar by stimulating either ear, such as cleaning the canal with a cotton swab. Unfortunately the cough persisted and repeated examinations and chest radiographs found no pathology to explain the symptoms.

He was admitted six months post-transplant for further investigation of his nagging cough. His symptoms were unchanged. He had no fever, chills, or chest pain. The physical examination was essentially normal with the exception of coughing paroxysms on otoscopic examination. Chest radiography was normal and flexible bronchoscopy was normal. A thorough otoscopic examination was performed by an otolaryngologist and was felt to be normal except that on examination of the right ear the patient went into a severe coughing paroxysm and laryngeal spasm. No hairs were in contact with the tympanic membrane. More than three years after surgery his symptoms remained unchanged. He would not comply with a trial of Tegretol.

\section{Discussion}

Mitempfindung (the German word for "with sympathy") has been described by several authors dating back to a paper by Stephen Hales in $1733^{1}$ and summarised in a recent publication by Schott. ${ }^{2}$ Briefly, it represents an unusual phenomenon of cutaneous referral whereby cutaneous stimulation at one site on the body elicits a simultaneous sensation at a remote site. In any one person the sites tend to be constant, ${ }^{3469}$ and it appears that a certain threshold of stimulus must be exceeded for the Mitempfindungen to occur. The referred sensation is usually more noxious than the initiating stimulus. The sites of the initiating stimulus and the referred sensation are almost always ipsilateral. The phenomenon may be congenital, appearing in otherwise healthy individuals, or acquired. ${ }^{2}$ When acquired there is usually a recent history of neurological damage in the area of referral. All reports so far describe such events in cutaneous distributions alone. ${ }^{1-9}$

The afferent arm of the cough reflex is normally subserved by the visceral sensory fibres from the larynx, trachea and bronchial tree supplied by the vagus nerve. General visceral sensory fibres from above the vocal folds are carried in the internal laryngeal nerve which joins the external laryngeal nerve to become the superior laryngeal nerve. General visceral sensory fibres from below the vocal folds ascend in the recurrent laryngeal nerve. Fibres of the superior and recurrent laryngeal nerves enter the inferior vagal ganglion en route to various centres induding the spinal nucleus of the trigeminal nerve and the dorsal motor nucleus of the vagus nerve. The cough reflex arc is initiated by the dorsal motor nucleus which sends impulses to the respiratory centre to coordinate forced expiration via the phrenic and intercostal nerves. ${ }^{78}$ Furthermore, general somatic afferent fibres of the auricular branch of the vagus nerve supply sensation to a small area of skin behind the ear 
and the posterior wall of the external auditory canal. ${ }^{7}$ These fibres end in the spinal nucleus of the trigeminal ganglion. It is recognised that in some individuals, "stimulation of the auricular branches of the vagus nerve in the external auditory meatus can cause reflex coughing, vomiting and even fainting through reflex activation of the dorsal vagal motor nucleus". Many clinicians have experienced this phenomenon in their patients during otoscopic examination, and such cases appear to be congenital or at least date to early life in the absence of known neurological disturbance.

Our case appears to represent an acquired aberrancy of sensory referral. Injury to the visceral sensory component of the right vagus nerve may have given rise to a Mitempfindunglike phenomenon in two of its branches. Stimulation in the cutaneous distribution now produces a referred irritating sensation in part of the general visceral sensory distribution which leads to coughing and occasionally gagging by activation of the cough reflex. The cutaneous stimulus probably activates the dorsal motor nucleus of the vagus nerve directly, producing a phenomenon akin to that described above during otoscopic examination.

As in the cases reported by Schott, the present case may have been acquired through neurological damage. ${ }^{2}$ During cardiac transplantation transection of the cardiac branches of the vagus nerve is unavoidable and the potential for damage to recurrent laryngeal nerve or tracheal sensory fibres exist. The transient hoarseness our patient experienced may have been the clinical manifestation of a partial injury to one of the recurrent laryngeal or tracheal sensory nerves thus setting the stage for acquired Mitempfindung to occur in its distribution. This could explain how cutaneous stimulation in the distribution of the right vagus nerve elicits a sensation of laryngeal irritation, causing the patient to cough. The latency period postoperatively seems too brief to postulate any form of aberrant regeneration. Alternatively there may have been a preferential injury to vagal fibres which normally serve to inhibit this referred sensation uncovering what is a normally suppressed reflex. Another possible explanation is that the vagal afferent itself may be in some way more irritable secondary to the injury with the aberrant referral resulting from a central mechanism triggered by the sensory afferents from the ear. As opposed to previous reports where the aberrant referral was from one cutaneous distribution to another, here there is referral from a cutaneous distribution to a visceral sensory distribution, both of which could be components of the same nerve (the vagus).

In his paper, Schott proposed the variable spinocervical tract as a site for the aberrant connections to exist. ${ }^{2}$ Evans ${ }^{5}$ proposed that the connections might lie in the thalamus or in suprathalamic structures. Whatever the proposed explanation, Mitempfindung represents a curious and interesting clinical phenomenon and neuroanatomical and physiological puzzle.

We thank DrW K C Morgan and Dr P W Pflugfelder for their clinical expertise.

1 Hales S. Statical essays, Vol 2. London: Hafner, 1733 $59-60$

2 Schott GD. Distant referral of cutaneous sensation (Mitempfindung). Brain 1988;111:1187-98.

3 Sterling P. Referred cutaneous sensation. Experimental Neurology 1973;1:451-6.

4 Bean WB. A curious double referral of mild pain produced by scratching the skin.: with a note on the gentle art of snoring. Trans Am Clin Climatological Association 1979; snoring. Trans

5 Evans PR. Referred itch (Mitempfindungen). BMF 1976; 2:839-41.

6 Richter CP. Mysterious form of referred sensation in man Proc Nat Acad Sci USA 1977;74(10):4702-5.

7 Truex RC, Carpenter MB. Neuroanatomy. Baltimore: Williams and Wilkins, 1969:321-6.

8 Wilson-Pauwels L, Akesson EJ, Stewart PA. Cranial nerves, anatomy and clinical comments. Toronto: BC Decker 1988:126-37.

9 Sinclair DC. The remote reference of pain aroused in the skin. Brain 1949;72:364-72. 